\title{
Altered Impedance of Ear Acupuncture Point MT2 in Breast Cancer Patients: A Preliminary Observation
}

\author{
Yine Hu, ${ }^{1}$ Huayuan Yang, ${ }^{1}$ Pin Wang, ${ }^{2}$ Tangyi Liu, ${ }^{1}$ and Wenchao Tang \\ ${ }^{1}$ Laboratory of Traditional Chinese Medicine Engineering, Shanghai University of Traditional Chinese Medicine, \\ 1200 Cailun Road, Shanghai 201203, China \\ ${ }^{2}$ Anhui University of Traditional Chinese Medicine, 103 Meishan Road, Hefei 230038, China
}

Correspondence should be addressed to Huayuan Yang; yanghy5921@163.com

Received 22 February 2015; Accepted 11 June 2015

Academic Editor: Francesco Pagnini

Copyright (C) 2015 Yine Hu et al. This is an open access article distributed under the Creative Commons Attribution License, which permits unrestricted use, distribution, and reproduction in any medium, provided the original work is properly cited.

\begin{abstract}
Skin impedance at acupuncture points (APs) has been used as a diagnostic aid for more than 50 years. In this study, we have a diagnostic tool (JXT-2008) to measure the skin impedance of ear APs of 30 breast cancer patients and the corresponding skin impedance of ear APs of 30 healthy humans, and then we compared these changes in ear AP impedance in breast cancer patients and healthy individuals.
\end{abstract}

\section{Introduction}

Acupuncture is a discipline in traditional Chinese medicine, which includes the practice of inserting fine needles into the skin at specific anatomical locations to stimulate certain points on the body. This therapeutic strategy has been practiced for thousands of years in the treatment of various human diseases. Acupuncture has already been adopted by a number of countries as a type of complementary or alternative medicine in the treatment of certain human diseases [1]. The general principle of acupuncture, based on traditional Chinese medicine, is that stimulation of particular acupuncture points (APs) can correct the imbalances of the flow of Qi through specific channels known as meridians [2]. Qi in Chinese traditional medicine is used to describe the refined nutritious substance that constitutes the human body and maintains life activities, such as Gu-Qi and nutrient Qi. Qi is also used to describe functions of Zang-Fu organs, such as heart Qi and liver Qi [3]. However, to date, scientific research has been unable to identify any histological or physiological associations between Qi and acupuncture points [4]. Acupuncture is specifically useful in the treatment of autonomic dysfunction, neurological conditions (such as migraine or pain), cardiovascular diseases, pulmonary diseases (such as asthma), drug abuse, psychological disorders, and others [5]. Thus, novel stimulation techniques and the discovery of new acupuncture points could lead to better acceptance by patients and more effective treatment of human diseases. To this end, a Japanese researcher claimed that the presence of low skin impedance at acupuncture points is better than that of the non-APs [6]. Thereafter, an instrument was developed to detect electric reaction in skin as a diagnostic aid in Germany [7]. Earlier studies on the skin impedance were particularly useful in the diagnosis and treatment of different diseases. Studies have shown that APs have been shown to have lower skin impedance than the non-APs in the healthy individuals [8-10] and therefore changes in skin impedance at APs can be of diagnostic significance. However, another study showed that skin impedance at APs can either be lower or higher compared to the surrounding skin area in the healthy individuals; thus, electrical skin resistance measurement may not be used for acupuncture point localization or diagnostic purposes [11]. This observation could be due to the fact that these studies included healthy individuals only. Another study indeed reported that there was no difference between APs and non-APs in healthy individuals and changes may occur during illness [12]. Thus, we assessed and detected the skin impedance between the healthy individuals and the cancer patients in this study. However, according to traditional Chinese medicine, acupuncture meridians are 
believed to constitute channels for Qi flow connecting the surface of the body to internal organs. The theory regarding acupuncture meridians as "transmission lines" suggested that meridians may represent networks of preferential electrical current flow [13].

Study on the skin impedance in the ear's APs was conducted as an early diagnosis strategy for the detection of certain cancer in patients. In the presence of benign or malignant tumor in the body, the meridians may sense and react to the meeting point of the body surface and internal organs, and then a series of changes in biological and physical activities will appear on the surface of the ear [14]. A study investigating AP reaction in a total of 745 patients with diseases in the digestive system, respiratory system, and cardiovascular system diagnosed by western medicine revealed positive association between diagnoses made by western medicine and APs [15]. The purpose of this study was to use a nonlinear detecting method to detect impedance in different ear's APs, to observe their basic features, and finally to compare LIA, LDA, RIA, and RDA to determine skin resistance. In addition, we also assessed whether ear skin impedance at APs can be used as a diagnostic tool for breast cancer. The observation will provide evidence on whether there are differences in skin impedances at APs in the same ear between breast cancer patients and healthy subjects.

\section{Materials and Methods}

2.1. Experimental Device. To detect changes in impedance at APs on the ear for the detection of breast cancer, we utilized the skin impedance detection system (model: JXT-2008) that was developed by Shanghai University of Traditional Chinese Medicine (Shanghai, China). A sensitive skin impedance detection-analysis system was used and internal design principles are shown in Figure 1.

The impedance at ear APs was measured after ensuring that the patients have established ground contact and using the device (i.e., JXT-2008) the patients were provided with continuous current with increasing intensity from $0 \mu \mathrm{A}$ to $20 \mu \mathrm{A}$ and then returning back from $20 \mu \mathrm{A}$ to $0 \mu \mathrm{A}$. This enabled the measurement of current-voltage characteristics curve on the surface of the human body. The system consists of 89C51 single-chip microcomputer (SCM), a digital-analog component (DAC), an analog-digital component (ADC), a constant current source component (CCS), a voltage follower, an attenuator, and two probes. The SCM was connected to a personal computer through USB data line. The hardware and software design of this skin impedance detecting instrument is illustrated in Figures 1(a) and 1(b), respectively.

2.2. Study Subjects. A total of 60 subjects (30 breast cancer patients and 30 healthy control individuals) were recruited from Longhua Hospital and Shanghai University of TCM (Shanghai, China) between August 2007 and January 2008. The 30 breast cancer patients recruited were all female aged between 23 and 45 years (with mean age of $34.6 \pm 12.14$ years). This study was approved by the Ethics Committee of
Shanghai Society and all participants signed an informed consent to participate in this study.

2.2.1. Inclusion Criteria. Inclusion criteria included (1) patients who were diagnosed with breast cancer histologically and (2) patients who had undergone complete radiotherapy and chemotherapy.

2.2.2. Exclusion Criteria. Exclusion criteria included patients who have any serious cardiovascular and cerebrovascular diseases, diseases of the blood system, serious damage to the liver and kidney functions, mental illness, or other cancers.

All participants completed quality of life questionnaires or psychiatric evaluation. Furthermore, 30 healthy controls were also recruited from Shanghai University of TCM (Shanghai, China) and these female volunteers were between 18 and 38 years (with mean age of $28.3 \pm 8.54$ years). They were all "healthy" as defined by normal body temperature and with no known autonomic nervous system dysfunction, coronary heart disease, systemic disease, cancer, or skin disease [16].

Ear APs Selection and Localization. A previous study demonstrated acupuncture treatment using the ear APs in the management of breast hyperplasia, and the ear APs were TF4 (Shenmen), BP-B3 (Ruxian), CO18 (Neifenmi), and AT4 (Pizhixia) [17]. In addition, the endocrine-related APs TG2p (Shenshangxian) [18] were also included in the current study. Moreover, clinical experience indicates that some specific area on the ear APs can provide a reference for the diagnosis of malignancies and these APs were named as MT1 (malignant tumor 1), MT2, and MT3, while MT2 is more closely associated with the diagnosis of malignancies [19]. Together, this study included the following ear APs (i.e., the bilateral CO18 (Neifenmi), AT4 (Pizhixia), TG2p (Shenshangxian), BP-B3 (Ruxian), and MT2 (malignant tumor 2)), which are more related to breast diseases. The localization of these ear APs was detected by GB/T13734-2008 nomenclature and location of ear points (Figure 2).

Detection Methods. The experiment was performed under quiet, controlled conditions with room temperature ranging between 20 and $26^{\circ} \mathrm{C}$ with a relative humidity of $50 \%$ to $75 \%$. All subjects were asked to arrive at the laboratory more than 15 minutes prior to the experiment and sat quietly to relax their muscles. The skin impedance was measured after the ear skin was cleaned with $75 \%$ alcohol for 30 seconds. The detecting probe was placed on the points with $75 \mathrm{~g} / \mathrm{mm}^{2}$ pressure with a detection angle of 90 degrees for a total scanning time of 20 seconds. The scanning electrical current was increased steadily and linearly from $0 \mu \mathrm{A}$ to $20 \mu \mathrm{A}$ in the first 10 seconds and was decreased steadily from $20 \mu \mathrm{A}$ to $0 \mu \mathrm{A}$ in the second 10 seconds. Measurements at every APs were recorded once every 3 seconds and the measurements cycle was repeated three times. Data were summarized as mean \pm SD.

Observation Index. The quantitative index of the skin impedance of APs included Left Increase Area (LIA), Right 


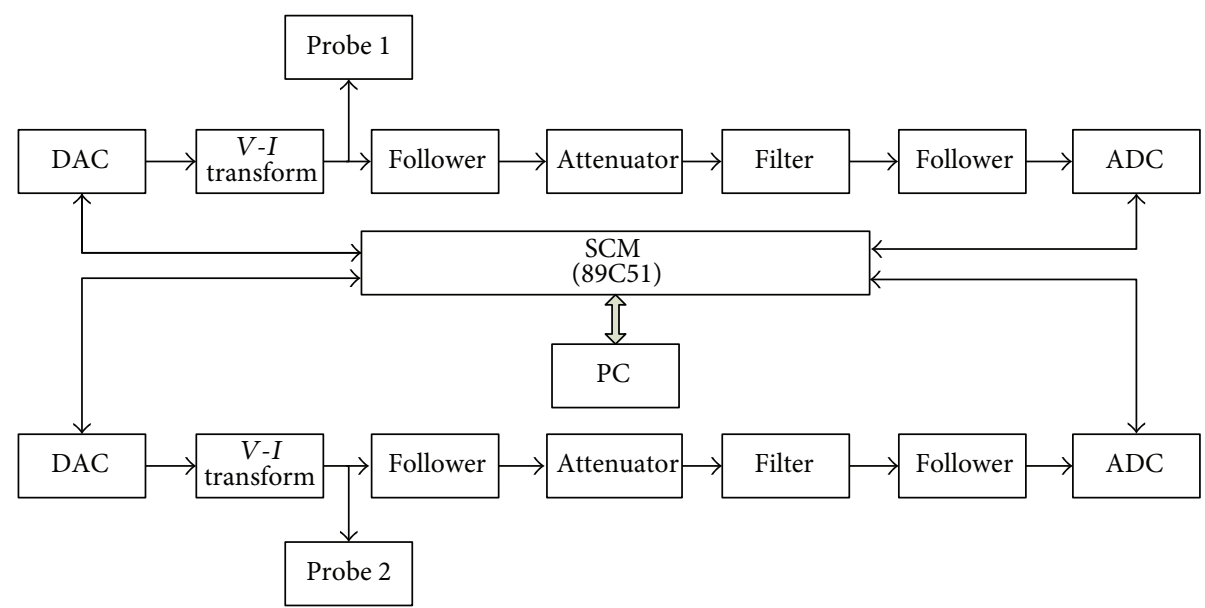

(a)
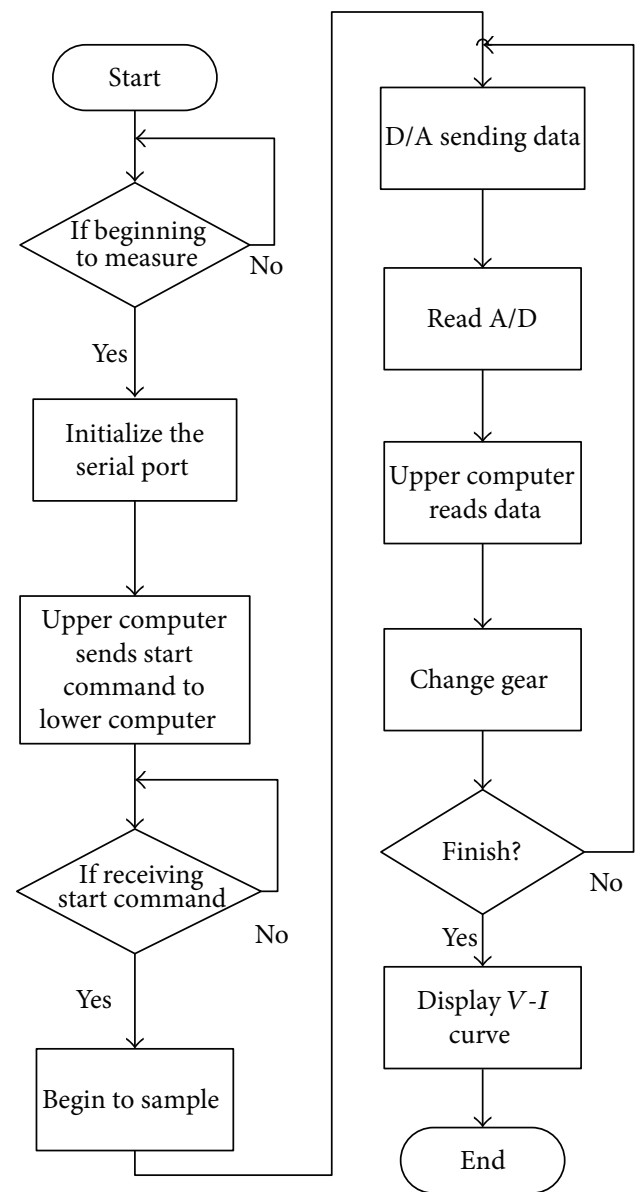

(b)

FIGURE 1: Illustration of our measurement device. (a) Hardware schematic diagram of the skin impedance detection system (JXT-2008). (b) Software schematic diagram of the skin impedance detection system (JXT-2008).

Increase Area (RIA), Left Decrease Area (LDA), and Right Decrease Area (RDA). Increase Area (IA) is referred to as the area comprised of the current-voltage curve (currents intensity was increased from $0 \mu \mathrm{A}$ to $20 \mu \mathrm{A}$ ) and abscissa, which was calculated by integral calculus. LIA refers to the left AP of two bilateral symmetrical APs, and RIA refers to the right one. When currents intensity was reversed from $20 \mu \mathrm{A}$ to $0 \mu \mathrm{A}$, the corresponding parameters (LDA and $\mathrm{RDA}$ ) were 


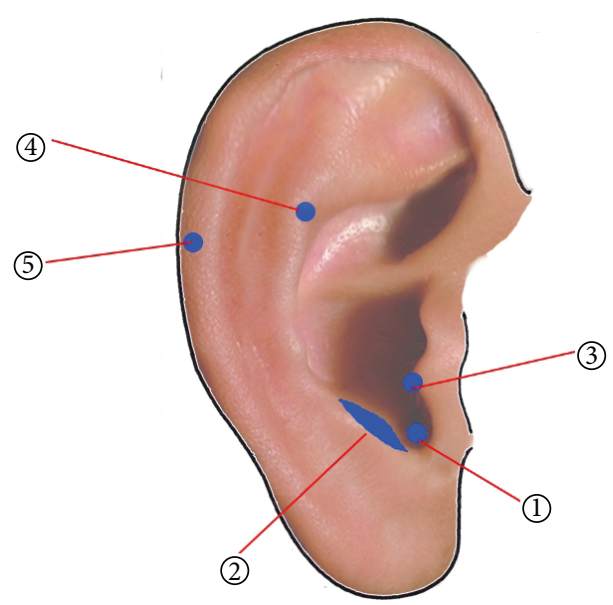

Figure 2: Ear APs location. (1) CO18 (Neifenmi); (2) AT4 (Pizhixia); (3) TG2p (Shenshangxian); (4) BP-B3 (Ruxian); and (5) MT2 (malignant tumor 2).

obtained. The area was calculated according to the following formula:

$$
\begin{gathered}
\text { Increase Area }=\int_{0}^{20} \mathrm{udI}, \\
\text { Decrease Area }=\int_{20}^{0} \mathrm{udI} .
\end{gathered}
$$

Statistical Analysis. Impedance in each ear's AP was measured 3 times and the data were summarized as mean \pm SD. Two independent samples' $t$-test was applied to compare the differences between healthy controls and breast cancer patients. The SPSS 16.0 statistical software (SPSS, Chicago, IL) was used for statistical analysis and $P$ value less than 0.05 was considered to be statistically significant.

\section{Results}

A total of 10 APs (i.e., bilateral CO18 (Neifenmi), AT4 (Pizhixia), TG2p (Shenshangxian), BP-B3 (Ruxian), and MT2 (malignant tumor 2)) were measured in 30 healthy individuals (the control group) and 30 breast cancer patients. The data are shown in Tables 1-5. Our results indicate that the skin impedance measured in LIA, LDA, RIA, and RDA of bilateral ear APs (AT4 and TG2p) was found to be higher in patients than those of the healthy individuals (control group); however, the differences were not statistically significant $(P>$ 0.05) (Tables 2 and 3). The AP spots of CO18 and BP-B3 recorded similar results; that is, at ear APs, CO18 and BP$\mathrm{B} 3$, the impedances in LIA and LDA were higher in patients than those of the healthy individuals (control group), whereas the RIA and RDA were lower in patients than those of the healthy individuals (control group), but the differences were not statistically significant $(P>0.05)$ (Tables 1 and 4$)$. Interestingly, volt-ampere area at AP MT2 (malignant tumor $2)$ was significantly $(P<0.05)$ higher in patients than those of the healthy individuals. Skin impedance of the ear AP MT2 in breast cancer patients was significantly increased
TABLE 1: Comparison of LIA, LDA, RIA, and RDA between patients and controls in CO18.

\begin{tabular}{lccccc}
\hline Index & Groups & Number & Area $(\bar{X} \pm S)$ & $P$ value & $t$ value \\
\hline LIA & $\begin{array}{c}\text { Breast cancer } \\
\text { Control }\end{array}$ & 30 & $57.20 \pm 41.09$ & 0.39 & 0.58 \\
\hline LDA & $\begin{array}{c}\text { Breast cancer } \\
\text { Control }\end{array}$ & 30 & $52.04 \pm 26.03$ & & \\
\hline \multirow{2}{*}{ RIA } & Breast cancer & 30 & $45.30 \pm 22.39$ & 0.53 & 0.84 \\
& Control & 30 & $43.29 \pm 25.60$ & 0.33 & 0.59 \\
\multirow{2}{*}{ RDA } & $\begin{array}{c}\text { Breast cancer } \\
\text { Control }\end{array}$ & 30 & $36.16 \pm 15.66$ & 0.48 & 0.62 \\
& Cont & $39.23 \pm 22.24$ & &
\end{tabular}

TABLE 2: Comparison of LIA, LDA, RIA, and RDA between patients and controls in AT4.

\begin{tabular}{lccccc}
\hline Index & Groups & Number & Area $(\bar{X} \pm S)$ & $P$ value & $t$ value \\
\hline LIA & $\begin{array}{c}\text { Breast cancer } \\
\text { Control }\end{array}$ & 30 & $85.74 \pm 46.44$ & 0.64 & 0.46 \\
\hline LDA & $\begin{array}{c}\text { Breast cancer } \\
\text { Control }\end{array}$ & $\begin{array}{c}30 \\
30\end{array}$ & $\begin{array}{c}76.94 \pm 43.31 \\
70.84 \pm 32.89\end{array}$ & 0.82 & 0.62 \\
\hline \multirow{2}{*}{ RIA } & Breast cancer & 30 & $70.15 \pm 35.80$ & 0.65 & 0.26 \\
& Control & 30 & $67.87 \pm 33.04$ & & \\
\hline \multirow{2}{*}{ RDA } & $\begin{array}{c}\text { Breast cancer } \\
\text { Control }\end{array}$ & 30 & $62.31 \pm 31.24$ & 0.77 & 0.23 \\
\hline
\end{tabular}

TABLE 3: Comparison of LIA, LDA, RIA, and RDA between patients

\begin{tabular}{|c|c|c|c|c|c|}
\hline Index & Groups & Number & Area $(\bar{X} \pm S)$ & $P$ value & $t$ value \\
\hline \multirow{2}{*}{ LIA } & Breast cancer & 30 & $68.70 \pm 43.80$ & \multirow{2}{*}{0.49} & \multirow{2}{*}{1.06} \\
\hline & Control & 30 & $58.15 \pm 32.40$ & & \\
\hline \multirow{2}{*}{ LDA } & Breast cancer & 30 & $59.69 \pm 39.20$ & \multirow{2}{*}{0.42} & \multirow{2}{*}{1.10} \\
\hline & Control & 30 & $49.96 \pm 28.39$ & & \\
\hline \multirow{2}{*}{ RIA } & Breast cancer & 30 & $57.86 \pm 39.19$ & \multirow{2}{*}{0.43} & \multirow{2}{*}{0.43} \\
\hline & Control & 30 & $54.03 \pm 28.13$ & & \\
\hline \multirow{2}{*}{$\mathrm{RDA}$} & Breast cancer & 30 & $50.08 \pm 33.65$ & \multirow{2}{*}{0.38} & \multirow{2}{*}{0.35} \\
\hline & Control & 30 & $47.37 \pm 24.58$ & & \\
\hline
\end{tabular}
and controls in TG2p.

$($ LIA, $P$ value $=0.0047 ;$ LDA, $P$ value $=0.0028 ;$ RIA, $P$ value $=0.0030 ;$ RDA, $P$ value $=0.0015)$ as listed in Table 5 . Statistical analyses indicate that all detected ear APs had nonlinear characteristics in both healthy control individuals and breast cancer patients. The skin impedance at other ear APs (i.e., CO18, AT4, TG2p, and BP-B3) was also altered in breast cancer patients, but the changes were not statistically significant compared to the healthy controls $(P>0.05)$.

\section{Discussion}

In the current study, we measured the ear APs impedance in breast cancer patients and healthy controls and found that the ear APs impedance in breast cancer patients can be either lower or higher compared to that of the healthy controls. The alterations in ear APs impedance could aid in diagnosis of these patients. If the APs skin impedance was 
TABLE 4: Comparison of the LIA, LDA, RIA, and RDA between patients and controls in BP-B3.

\begin{tabular}{lccccc}
\hline Index & Groups & Number & Area $(\bar{X} \pm S)$ & $P$ value & $t$ value \\
\hline LIA & $\begin{array}{c}\text { Breast cancer } \\
\text { Control }\end{array}$ & 30 & $123.95 \pm 45.74$ & 0.11 & 1.58 \\
\hline LDA & Breast cancer & 30 & $105.34 \pm 45.55$ & & \\
& Control & 30 & $94.85 \pm 42.92$ & 0.20 & 1.29 \\
\hline \multirow{2}{*}{ RIA } & $\begin{array}{c}\text { Breast cancer } \\
\text { Control }\end{array}$ & 30 & $103.05 \pm 46.15$ & 0.77 & 0.28 \\
\hline \multirow{2}{*}{ RDA } & $\begin{array}{c}\text { Breast cancer } \\
\text { Control }\end{array}$ & 30 & $106.44 \pm 46.99$ & & \\
\hline
\end{tabular}

TABLE 5: Comparison of LIA, LDA, RIA, and RDA between patients and controls in MT2.

\begin{tabular}{|c|c|c|c|c|c|}
\hline Index & Groups & Number & Area $(\bar{X} \pm S)$ & $P$ values & $t$ values \\
\hline \multirow{2}{*}{ LIA } & BC patients & 30 & $79.64 \pm 47.79^{* *}$ & \multirow{2}{*}{0.0047} & \multirow{2}{*}{3.42} \\
\hline & Control & 30 & $47.36 \pm 19.77$ & & \\
\hline \multirow{2}{*}{ LDA } & BC patients & 30 & $70.47 \pm 43.45^{* *}$ & \multirow{2}{*}{0.0028} & \multirow{2}{*}{3.32} \\
\hline & Control & 30 & $41.84 \pm 18.60$ & & \\
\hline \multirow{2}{*}{ RIA } & BC patients & 30 & $81.40 \pm 46.91^{* *}$ & \multirow{2}{*}{0.0030} & \multirow{2}{*}{3.31} \\
\hline & Control & 30 & $49.50 \pm 24.18$ & & \\
\hline \multirow{2}{*}{$\mathrm{RDA}$} & BC patients & 30 & $71.12 \pm 41.20^{* *}$ & \multirow{2}{*}{0.0015} & \multirow{2}{*}{3.52} \\
\hline & Control & 30 & $41.81 \pm 19.49$ & & \\
\hline
\end{tabular}

${ }^{* *} P<0.01$.

regarded as a "point" that changes in time and space, then the volt-ampere area (i.e., LIA, LDA, RIA, and RDA) reflects comprehensive dynamic resistance between APs. Specifically, we identified that in LIA, LDA, RIA, and RDA and there was a statistically significant difference in bilateral ear AP MT2 between breast cancer patients and healthy individuals. Scientific evidence suggested that ear skin impedance at the APs is electrically distinct from non-AP sites and that changes in ear skin AP impedance could be of substantial diagnostic, therapeutic, and research significance. Indeed, in China and certain western countries, different researchers have tried to demonstrate a difference between the skin impedance at AP and the surrounding impedance and showed that APs had lower skin resistance than nearby non-AP sites [20,21], which is useful in localization of APs. Moreover, another study showed that skin impedance at AP sites could be either lower or, in contrast to common belief, higher compared to the surrounding area and that this phenomenon had a high shortterm and a low long-term reproducibility. It is important to note that these data were obtained from healthy individuals, and there were no data on skin impedance at AP sites in individuals suffering from a disease.

Evidence based on previous studies indicated that in certain diseases higher or lower resistance of skin impedance at APs was associated with clinically relevant APs; for example, in stomach diseases, the APs with differential impedance were observed in ST36 (Zusanli), RN12 (Zhongwan), and BL21 (Weishu), but not at other clinically unrelated APs or non-AP sites in the same patients $[22,23]$. Observations from the current study were consistent with a previous study that higher value at the volt-ampere area indicated greater impedance and the nonlinear characteristics of the volt-ampere curve [24]. Our earlier studies with this skin impedance measuring device reported skin impedances in stroke patients were higher than that of the healthy controls $[25,26]$.

As a result, skin impedance value may be used in the early aid diagnosis of breast cancer. It was suggested that its changes could be due to loss of cellular cohesion and increase in the extracellular space in skin tumors [27]. According to Sugar's report, the extracellular spaces during inflammation also increased, but the increase was significantly less than in cancerous tissues. Thus, it is possible that skin impedance value may be appropriate for the early detection of different pathologies. In our current study, we selected five clinically relevant APs (bilateral CO18, AT4, TG2p, BP-B3, and MT2). We found that two clinically related APs (bilateral MT2) of breast cancer patients had higher skin impedance than that of the healthy controls, consistent with a previous study that characterized a slight increase of the normalized resistance in breast cancer patients versus healthy women [28]. However, our data was on impedance at APs on ear skin, based on the traditional acupuncture meridians theory of traditional Chinese medicine. An earlier study using impedance measurement system reported by Keshtkar et al. also showed difference in impedance between normal and malignant bladder tissue [29] and the resistivity of malignant tissues was higher than that of the nonmalignant tissues. However, our current data on ear APs (i.e., CO18, AT4, TG2p, and BPB3) were not similar to those reported by Keshtkar et al. The skin impedance values on the left or the right APs often vary, the skin impedance value of the same side of viscera that suffered from an illness was much lower than that of the side that was healthy, and this was very meaningful to locate the lesion in human body, which is in accordance with the meridian balance theory. Use of the APs as a diagnostic tool is carried out based on the understanding that APs have an association with the internal organs of the human body. The modern electronic technology combined with traditional Chinese medicine diagnostic method was used widely in different types of scientific research $[30,31]$. The skin impedance measurement of the APs gave an enormous impetus to development of diagnostic tools in traditional Chinese medicine, which can help to show certain information about the pathological changes more deeply and comprehensively.

To the best of our knowledge, there are no studies in scientific literature describing skin impedance at the same ear APs between breast cancer patients and healthy controls. We found that ear APs of breast cancer patients and healthy individuals might possess different specific electrical properties. Since the majority of the measured ear APs did not show higher skin impedance, it cannot be concluded from our data that the skin impedance measurements of ear APs (i.e., CO18, AT4, TG2p, and BP-B3) can be used for diagnostic purpose. However, the ear AP MT2 site could be used as diagnostic pointer in diagnosis of breast cancer in patients. Nevertheless, a possible limitation of this study is that it is based on a single population and the observations made may not be applicable for another population. The impedance did 
differ significantly between older and younger people in both the control and patient groups. However, it is important to note that the age of the study population was 45 years or younger.

\section{Conclusions}

The current study demonstrates that the skin impedance of breast cancer patients was lower than that of healthy controls only at one AP (MT2) in the ear and there were no differences in the impedance at other ear APs between the groups. Further studies involving a larger cohort are necessary to verify the current observation and investigate its accuracy in the detection of early stage breast cancer and staging of the disease.

$\begin{array}{ll}\text { Abbreviations } \\ \text { Bilateral CO18: } & \text { Neifenmi } \\ \text { AT4: } & \text { Pizhixia } \\ \text { TG2p: } & \text { Shenshangxian } \\ \text { BP-B3: } & \text { Ruxian } \\ \text { MT2: } & \text { Malignant tumor 2 } \\ \text { APs: } & \text { Acupuncture points } \\ \text { JXT-2008: } & \text { JinXueTanCe-2008. }\end{array}$

\section{Conflict of Interests}

The authors declared that there is no conflict of interests involved in this work.

\section{Authors' Contribution}

Yine $\mathrm{Hu}$ carried out the study design, participated in the sequence alignment, and drafted the paper. Wenchao Tang carried out the clinical trials. Pin Wang participated in measuring instrument hardware design and implementation. Tangyi Liu participated in the design of the study and performed the statistical analysis. Huayuan Yang conceived of the study and participated in its design and coordination and helped to draft the paper. All authors read and approved the final paper.

\section{Acknowledgments}

The authors would like to thank all the participants in this study. This study was supported in part by a grant from the National Natural Science Foundation of China (no. 81072880).

\section{References}

[1] World Health Organization, Acupuncture: Review and Analysis of Reports on Controlled Clinical Trials, World Health Organization, Geneva, Switzerland, 2003.

[2] X. Sheng, Meridian and Acupuncture, Chinese Medicine Press, Beijing, China, 2003.

[3] W. Yao, H. Yang, and G. Ding, "Mechanisms of Qi-blood circulation and Qi deficiency syndrome in view of blood and interstitial fluid circulation," Journal of Traditional Chinese Medicine, vol. 33, no. 4, pp. 538-544, 2013.

[4] M. Bauer, "The final days of traditional beliefs?-part one," Chinese Medicine Times, vol. 1, no. 4, 2006.

[5] A. Sato, P. Li, and J. L. Campbell, Acupuncture: Is There a Physiological Basis? Elsevier Science, Amsterdam, The Netherlands, 2002.

[6] Y. Nakatani, "On the nature of the acupuncture points and meridias," Journal of the Japan Society for Oriental Medicine, vol. 3, pp. 39-49, 1953.

[7] R. Voll, "Twenty years of electroacupuncture diagnosis in Germany: a progress report," American Journal of Acupuncture, vol. 3, pp. 7-17, 1975.

[8] A. C. Ahn, J. Wu, G. J. Badger, R. Hammerschlag, and H. M. Langevin, "Electrical impedance along connective tissue planes associated with acupuncture meridians," BMC Complementary and Alternative Medicine, vol. 5, no. 1, article 10, 2005.

[9] A. C. Ahn, A. P. Colbert, B. J. Anderson et al., "Electrical properties of acupuncture points and meridians: a systematic review," Bioelectromagnetics, vol. 29, no. 4, pp. 245-256, 2008.

[10] A. P. Colbert, R. Hammerschlag, M. Aickin, and J. McNames, "Reliability of the prognos electrodermal device for measurements of electrical skin resistance at acupuncture points," Journal of Alternative \& Complementary Medicine, vol. 10, no. 4, pp. 610-616, 2004.

[11] S. Kramer, K. Winterhalter, G. Schober et al., "Characteristics of electrical skin resistance at acupuncture points in healthy humans," Journal of Alternative and Complementary Medicine, vol. 15, no. 5, pp. 495-500, 2009.

[12] S. Pearson, A. P. Colbert, J. McNames, M. Baumgartner, and R. Hammerschlag, "Electrical skin impedance at acupuncture points," The Journal of Alternative and Complementary Medicine, vol. 13, no. 4, pp. 409-418, 2007.

[13] K.-T. Yung, "A birdcage model for the Chinese meridian system. Part I. A channel as a transmission line," The American Journal of Chinese Medicine, vol. 32, no. 5, pp. 815-828, 2004.

[14] Y. Wang and Y. Zhai, "The clinical application value of the HX tumor measuring instrument," Medical Equipment, vol. 12, pp. 18-20, 1999.

[15] Y. Zhang and Y. Yang, "The acupoint reaction significance in cancer screening and cancer diagnosis," Journal of Clinical Acupuncture, vol. 12, pp. 52-53, 1996.

[16] H. M. Langevin, D. L. Churchill, J. R. Fox, G. J. Badger, B. S. Garra, and M. H. Krag, "Biomechanical response to acupuncture needling in humans," Journal of Applied Physiology, vol. 91, no. 6, pp. 2471-2478, 2001.

[17] B. Shen, "Ear therapy treatment of 112 cases of breast hyperplasia," Shanxi Journal of Traditional Chinese Medicine, vol. 24, p. 649, 2003.

[18] M. Valenzuela and T. B. Julian, "Neo-adjuvant hormonal therapy," The Breast Journal, vol. 14, no. 3, pp. 279-283, 2008.

[19] Y. Chang, "The nomenclature and location of the qualitative Ear APs about tumor," Journal of Clinical Acupuncture and Moxibustion, vol. 192, pp. 24-25, 1990.

[20] A. Mayer-Gindner, A. Lek-Uthai, O. Abdallah, and A. Bolz, "Newly explored electrical properties of normal skin and special skin sites," Biomedical Engineering, vol. 49, no. 5, pp. 117-124, 2004.

[21] A. P. Colbert, J. Yun, A. Larsen, T. Edinger, W. L. Gregory, and T. Thong, "Skin impedance measurements for acupuncture research: development of a continuous recording system," 
Evidence-based Complementary and Alternative Medicine, vol. 5, no. 4, pp. 443-450, 2008.

[22] J. Krop, G. T. Lewith, W. Gziut, and C. Radulescu, "A double blind, randomized, controlled investigation of electrodermal testing in the diagnosis of allergies," The Journal of Alternative and Complementary Medicine, vol. 3, no. 3, pp. 241-248, 1997.

[23] A. Margolin, S. K. Avants, S. Birch, C. X. Falk, and H. D. Kleber, "Methodological investigations for a multisite trial of auricular acupuncture for cocaine addiction: a study of active and control auricular zones," Journal of Substance Abuse Treatment, vol. 13, no. 6, pp. 471-481, 1996.

[24] J. Wei, Y. Zhou, X. Shen et al., "Circadian difference of voltampere characteristics of acupointsdaling (PC7)," Journal of the ACM, vol. 13, p. 903, 2007.

[25] H. Yang, J. Xia, and X. Gu, "The measurement system of meridian skin resistance dynamic parameter and its application," Beijing Biomedical Engineering, vol. 16, pp. 41-43, 1997.

[26] P. Wang, H.-Y. Yang, and Y.-Q. Wang, "Impedance characteristics of ear acupoints in identifying excess or deficiency syndrome of stroke," Journal of Chinese Integrative Medicine, vol. 8, no. 6, pp. 525-529, 2010.

[27] F. Sugár, "An electron microscopic study of early invasive growth in human skin tumours and laryngeal carcinoma," European Journal of Cancer, vol. 4, no. 1, pp. 33-38, 1968.

[28] T. MaŁecka-Massalska, K. Chara, A. Smolen, A. Kurylcio, W. Polkowski, and K. Lupa-Zatwarnicka, "Bioimpedance vector pattern in women with breast cancer detected by bioelectric impedance vector analysis. Preliminary observations," Annals of Agricultural and Environmental Medicine, vol. 19, no. 4, pp. 697-700, 2012.

[29] A. Keshtkar, Z. Salehnia, A. Keshtkar, and B. Shokouhi, "Bladder cancer detection using electrical impedance technique (Tabriz Mark 1)," Pathology Research International, vol. 2012, Article ID 470101, 5 pages, 2012.

[30] A. C. Y. Tang, J. W. Y. Chung, and T. K. S. Wong, "Validation of a novel traditional Chinese medicine pulse diagnostic model using an artificial neural network," Evidence-based Complementary and Alternative Medicine, vol. 2012, Article ID 685094, 7 pages, 2012.

[31] H. Kang, Y. Zhao, C. Li et al., "Integrating clinical indexes into four-diagnostic information contributes to the Traditional Chinese Medicine (TCM) syndrome diagnosis of chronic hepatitis B," Scientific Reports, vol. 5, article 9395, 2015. 


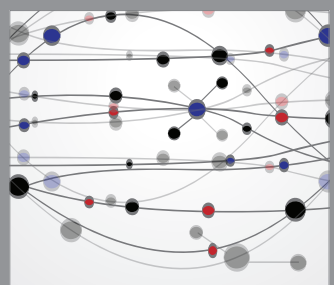

The Scientific World Journal
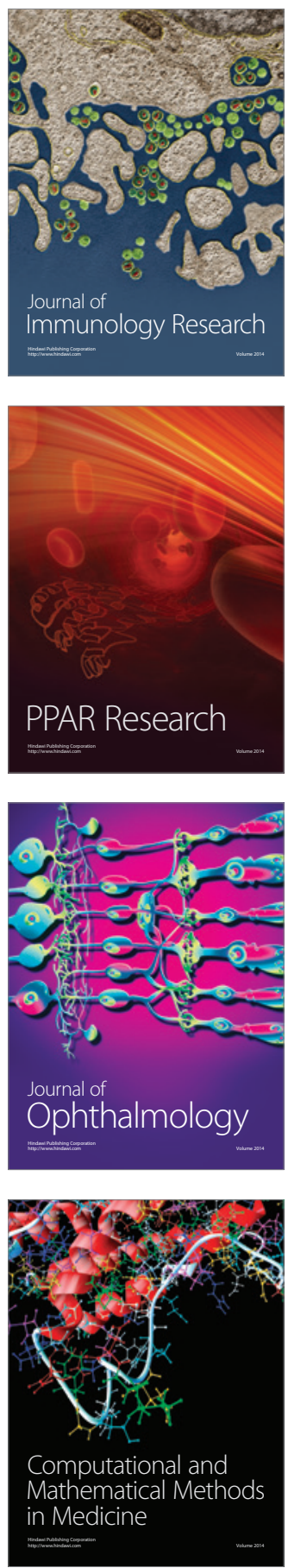

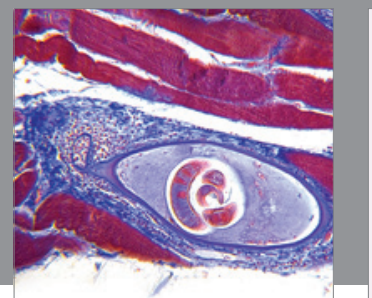

Gastroenterology

Research and Practice
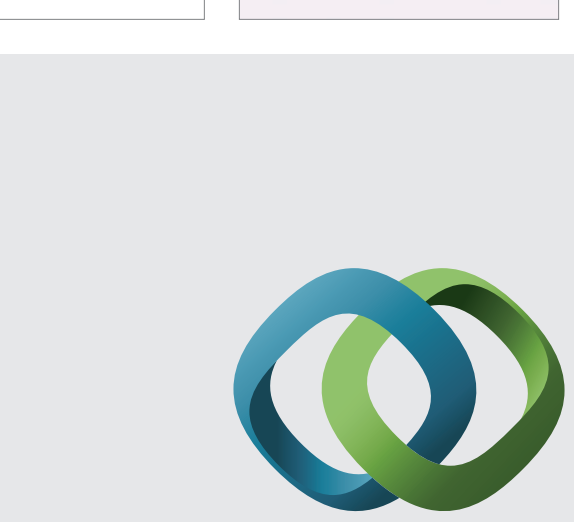

\section{Hindawi}

Submit your manuscripts at

http://www.hindawi.com
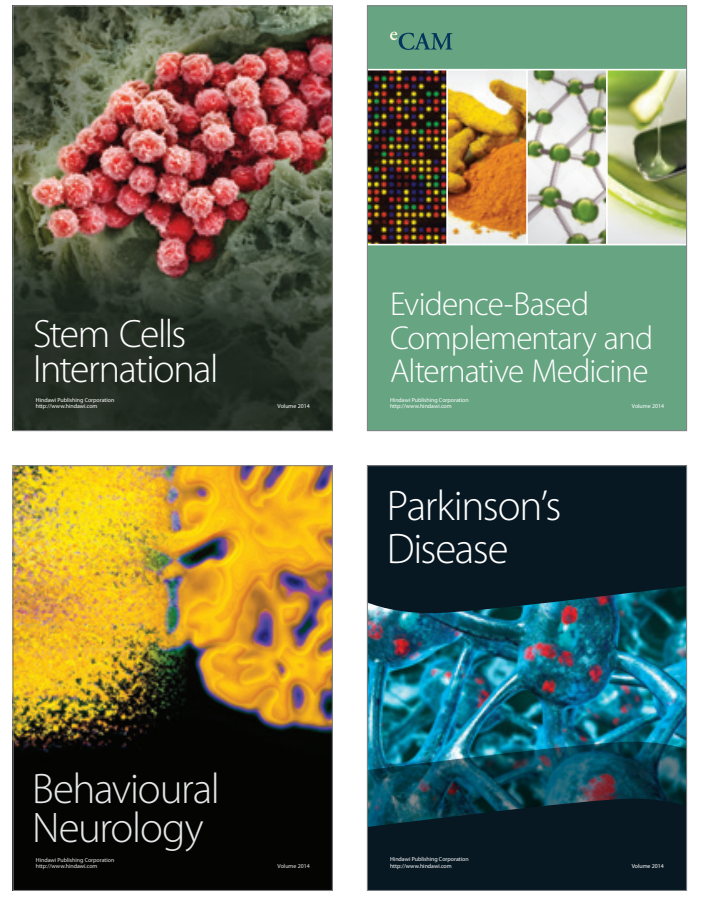
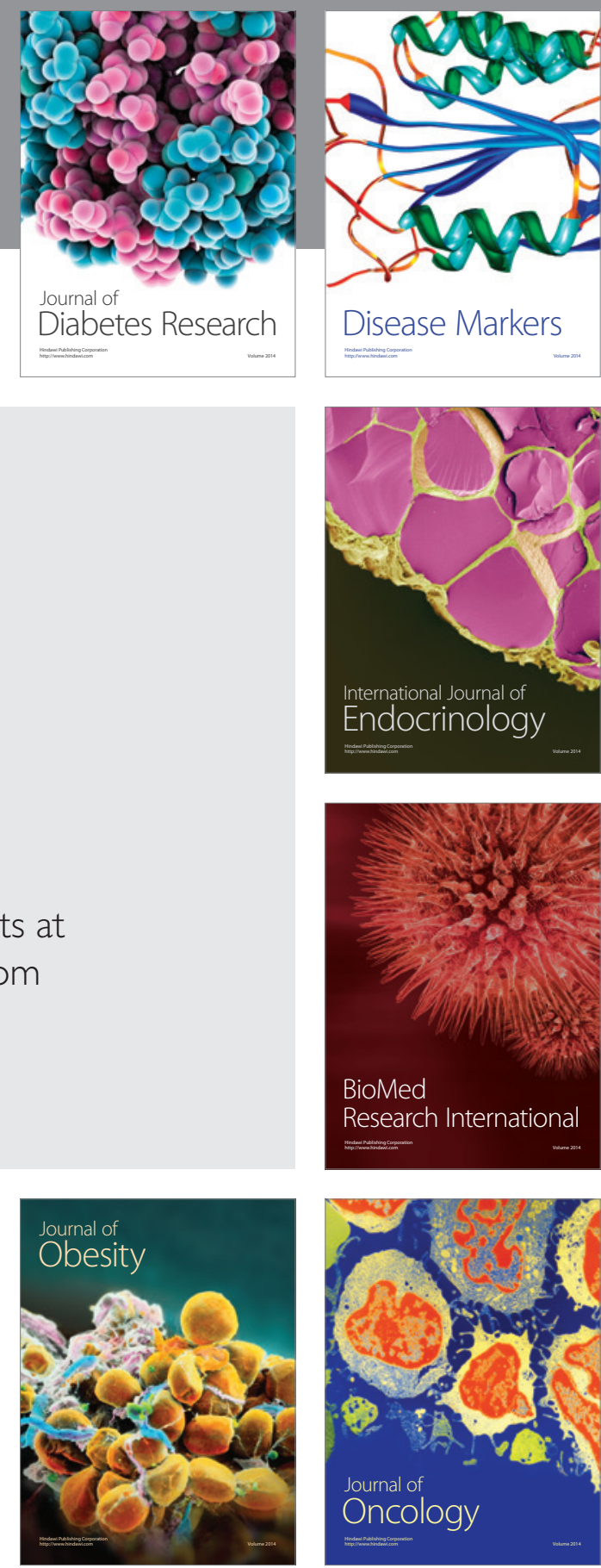

Disease Markers
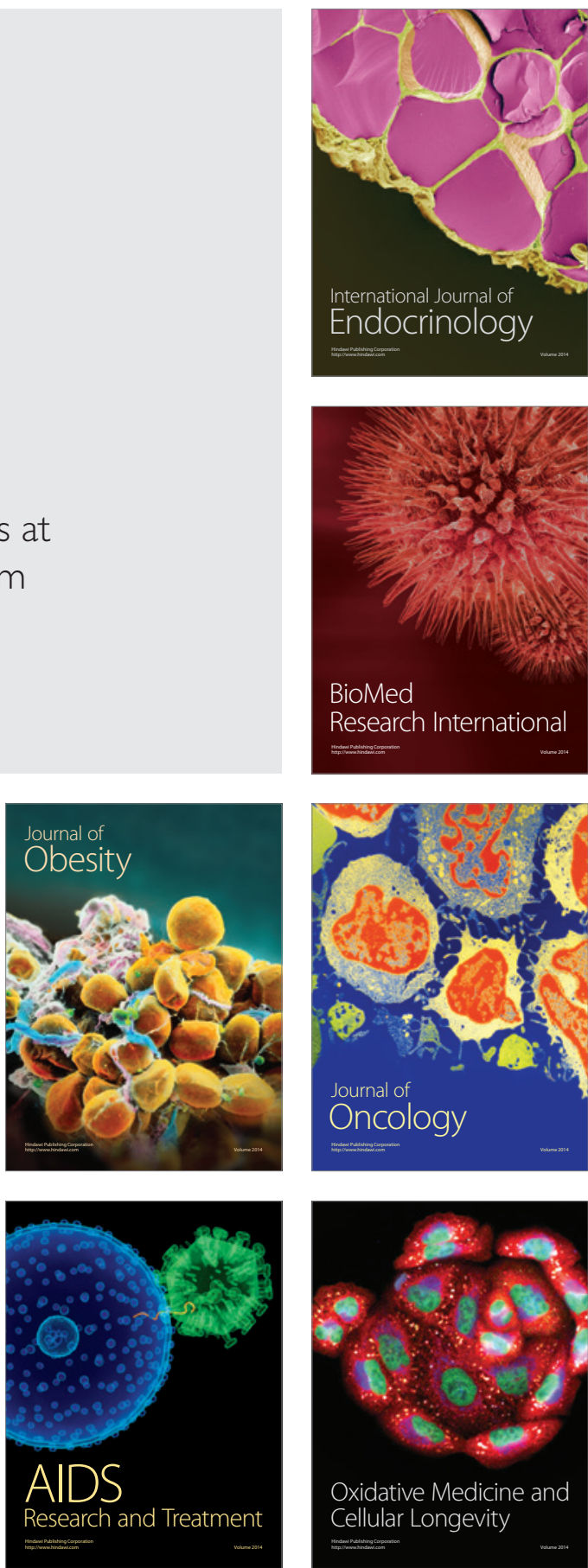